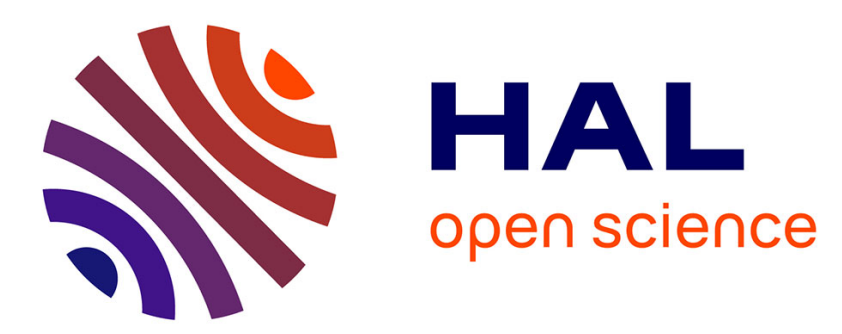

\title{
Stochastic modelling of tree annual shoot dynamics
}

\author{
Reffye, Kang, Hua, Auclair
}

\section{To cite this version:}

Reffye, Kang, Hua, Auclair. Stochastic modelling of tree annual shoot dynamics. Annals of Forest Science, 2012, 69 (2), pp.153-165. 10.1007/s13595-011-0151-6 . hal-00930722

\section{HAL Id: hal-00930722 \\ https://hal.science/hal-00930722}

Submitted on 1 Jan 2012

HAL is a multi-disciplinary open access archive for the deposit and dissemination of scientific research documents, whether they are published or not. The documents may come from teaching and research institutions in France or abroad, or from public or private research centers.

Copyright

L'archive ouverte pluridisciplinaire HAL, est destinée au dépôt et à la diffusion de documents scientifiques de niveau recherche, publiés ou non, émanant des établissements d'enseignement et de recherche français ou étrangers, des laboratoires publics ou privés. 


\title{
Stochastic modelling of tree annual shoot dynamics
}

\author{
Philippe de Reffye • MengZhen Kang • Jing Hua • \\ Daniel Auclair
}

Received: 11 February 2011 / Accepted: 18 October 2011 / Published online: 18 November 2011

(C) INRA and Springer-Verlag France 2011

\begin{abstract}
- Context Modelling annual shoot development processes is a key step towards functional-structural modelling of trees. Various patterns of meristem activity can be distinguished in tree shoots, with active periods of phytomer production followed by rest periods. This approach has seldom been integrated in functional-structural tree models. - Aims This paper presents theoretical research work on modelling and computation of the dynamics of tree annual shoots using stochastic processes with various development
\end{abstract}

\section{Handling Editor: Erwin Dreyer}

Contribution of the co-authors Philippe de Reffye: developed the mathematical equations related to renewal theory and supervised the work. MengZhen Kang: developed the simulation method of the model applied to tree structure.

This work is part of the $\mathrm{PhD}$ project of Jing Hua, who developed the computer programmes for the model implementation.

Daniel Auclair: was in charge of the model applications and co-supervised the work. He edited the English version of the manuscript.

\section{P. de Reffye \\ CIRAD, UMR AMAP, \\ Montpellier 34000, France}

\section{M.Z. Kang ( $\square)$}

State Key Laboratory of Management and Control for Complex Systems, LIAMA, NLPR, Institute of Automation,

Chinese Academy of Sciences,

Beijing 100190, China

e-mail: mengzhen.kang@ia.ac.cn

\section{J. Hua}

Chinese Academy of Sciences; LIAMA; NLPR,

Institute of Automation,

Beijing 100190, China

D. Auclair

INRA, UMR AMAP,

Montpellier 34000, France patterns: continuous or rhythmic, monocyclic or polycyclic, "seasonal" or "a-seasonal", with preformation or neoformation produced from meristem functioning.

- Methods The renewal theory is used to compute stochastic aspects of phytomer production, resulting from meristem extension or rest periods and meristem mortality.

- Results Continuous development can be modelled with a Bernoulli process, while rhythmic development is modelled by alternation between extension and rest periods, the duration of each period following specific distributions.

- Conclusion The application of such stochastic modelling is the estimation of organ production during tree development as a component of the demand in functionalarchitectural tree models, used for computing biomass production and partitioning.

Keywords Renewal theory - Architectural tree model · Meristem functioning $\cdot$ Polycyclism · Monocyclism · GreenLab

\section{Introduction}

Depending on their objectives, models of forest stand dynamics may account for individual tree structure or not (Kohyama et al. 2005; Pretzsch 2009; Feng et al. 2012). Schematically, stand-level models can be subdivided in "Empirical Forest Models", mostly used for forest management purposes, and "Process Based Models", which aim at assessing biomass production at the stand level, based on ecophysiological process, and these two types of models can be combined into "hybrid simulation models" (Mäkelä et al. 2000; Kimmins et al. 2008). Stand-level models generally include a very simple description of the plant 
structure: the trunk is described basically for diameter and height, and in some cases the shape of the living crown is included. Branch distribution along the stem is sometimes added, but is generally not linked to annual shoot development. Biomass can be allocated to compartments: crown, trunk, and root system; tree structure is rarely represented at the scale of individual shoots.

Nowadays, it is becoming increasingly important to take into account individual tree structure in forest management for different objectives including for example carbon allocation and sequestration (Melson et al. 2011), fuel description for fire risk assessment (Parsons et al. 2011), stand or landscape visualization (Griffon et al. 2011), or for calibrating models for remote sensing applications (Biliouris et al. 2009). With improvements in scientific knowledge and increased computing capacity, in conjunction with the requirement for more detailed representations, forest modellers seek to include some kind of representation of individual tree structure (Le Roux et al. 2001).

Individual tree models are generally classified into three main categories:

1. Morphological models aim at simulating tree structure without consideration of development processes (Kurth 1994). The main goal is to obtain realistic tree shapes to be used for example in computer graphics. In these models, the "virtual meristem" production is controlled by a set of rules that can lead to various branching patterns, while precise botanical knowledge on tree architecture is not necessary. Software like Xfrog (www.xfrog.com, Deussen and Lintermann 2005) and OnyxTree (www.onyxtree.com, Bosanac and Zanchi 2002) fulfil quite well this target.

2. Architectural models simulate the dynamics of development of the tree structure, which includes both topology and geometry, by integrating the architectural processes which influence the dynamics of the structure: endogenous processes inherent to each species and exogenous constraints exerted by the environment (Barthélémy et al. 1989). They are an extension of the initial architectural models described by Hallé et al. (1978). For example, AMAPsim (de Reffye et al. 1995; Barczi et al. 2008) considers architectural knowledge and represents bud functioning processes by stochastic variables. AMAPsim simulates the tree topological and geometrical structures, including those resulting from polycyclism; however, the alternation process of rest and extension periods of the meristems remains insufficiently represented.

3. Functional-structural plant models (FSPM) simulate plant development and growth regulated by physiological processes, generally considering source and sink compartments. Here, the meristem functioning generates the development of the plant structure by creating new organs (which play a role as source and/or sink), and expansion of organs by the incoming biomass generates tree growth (Vos et al. 2007). Such models are still an important challenge for research (De Jong and Da Silva 2010). In their recent review, Vos et al. (2010) discussed numerous examples of FSPMs, developed mostly for small plants: cereals, grasses, crop plants, and small trees (mainly fruit trees). Many are rule-based and use grammars such as Lsystems (Prusinkiewicz and Lindenmayer 1990). Few detailed FSPMs exist for forest trees because of both the structural complexity and the time required for simulations. LIGNUM (Perttunen et al. 1996) is an example of deterministic model, which simulates simple tree structures such as that of Pine: axes are considered as a stack of preformed growth units (GU) with immediate expansion. This is a relevant simplification for trees whose development (creation of new GUs) occurs in a short period (spring), while growth (extension, secondary growth, photosynthesis) lasts a long period (until autumn; Barthélémy and Caraglio 2007).

In the literature concerning FSPMs, the term "architecture" is often used alternatively to descriptive terms such as structure, morphology, or geometry, following the suggestion of Godin (2000) who considers architecture as an "individual description based on decomposition of the plant into components [...]". The description includes plant composition, geometrical properties, and topology (Vos et al. 2007). This definition however remains ambiguous in the sense that it is restricted to a description (which may be static or dynamic), but does not necessarily include the architecture development processes described in the botanical literature (Hallé et al. 1978; Barthélémy and Caraglio 2007). A fourth category of individual tree models could be defined as "Functional-Architectural Plant Models" (FAPM), which combine the architectural processes (endogenous and exogenous) of plant structure dynamics with physiological processes. Several FSPMs cited by Vos et al. (2010) include architectural processes for small plants. GreenLab (Yan et al. 2004) attempts to combine architectural development with physiological processes for trees. It is based on a similar paradigm to LIGNUM but includes more detailed architectural development simulated by stochastic processes similar to the AMAPsim architectural model (Barczi et al. 2008).

Both LIGNUM and the first versions of GreenLab were well adapted to trees which exhibit a short period of shoot expansion, where meristem development is immediate, issued from "preformation" with no "neoformation" (Barthélémy and Caraglio 2007, see Appendix 1). As a simplification, architectural development and biomass production can be decoupled: with an annual time step, these models account in 
a first stage for shoot extension and biomass production resulting from photosynthesis is modelled in a second stage on a static structure. This is not adapted to more complex cases where tree architectural development and biomass production occur simultaneously during long periods of time, for example where polycyclism, anticipated growth, or neoformation occurs as in Walnut (Sabatier and Barthélémy 2001), Poplar (Caraglio et al. 1990), or Elm (de Reffye et al. 1991a). Periods of extension and rest can occur successively between growth units but also within growth units. The present work addresses the general case where meristem activity may include periods of extension followed by rest periods and where photosynthetic activity occurs continuously (according to environmental conditions), independently of meristem activity. Instead of simulating the development of each organ individually, we use the renewal theory to propose a set of equations based on the meristem development processes. We have restricted the present work to individual shoot apical meristem dynamics, and the branching processes, addressed elsewhere (see Kang et al. 2008), will only be used here to illustrate the result of this work on whole tree architecture.

\section{Materials and methods}

\subsection{Botanical bases of plant architectural processes}

The study of plant architecture as a modern scientific discipline was initiated by Hallé et al. (1978) and has been recently reviewed by Barthélémy and Caraglio (2007), who described plant architecture as a dynamic process which is "the expression of an equilibrium between endogenous growth processes and exogenous constraints exerted by the environment". The particular processes relevant for the present study are described in Appendix 1: continuous and rhythmic development, pre- and neo-formation, mono-, and polycyclism. We distinguish "seasonal development" for regions with a marked seasonal difference (temperate regions or regions with dry and/or rainy seasons) and "a-seasonal development" for tropical or equatorial regions with no marked seasonal difference.

\subsection{Data used for examples of simulation}

The equations proposed here were developed on the basis of distributions observed on the original data published by de Reffye et al. (1991b), Barthélémy and Caraglio (2007), Mathieu et al. (2008), and de Reffye et al. (2008). Several typical examples of distributions and the corresponding Monte Carlo simulations are presented here to illustrate the consistency of the equations. Data on Bamboo from Dabadie et al. (1991) were used to illustrate the distribution of a number of phytomers observed on a plant with continuous development. Data on Hevea from de Reffye et al. (1991b) were used to illustrate the distribution of number of phytomers for "aseasonal" rhythmic shoot development. Observations from Sabatier and Barthélémy (2001) on Walnut were used to illustrate the distribution of number of GUs and phytomers for "seasonal" shoots with polyclism. To illustrate preformation followed immediately by neoformation with continuous extension, data on Japanese elm were provided by de Reffye et al. (1991a), and a final illustration of whole tree architecture is based on data on Populus from Caraglio et al. (1990).

\subsection{Initial developments of GreenLab}

GreenLab (GL) is a mathematical plant model that simulates interactions between plant architecture and function. A dual-scale automaton is used to simulate plant organogenesis from germination to maturity on the basis of organogenetic growth cycles that depend upon thermal time. Plant biomass production is computed from transpiration and distributed among expanding organs according to their relative demand (Yan et al. 2004; Wang et al. 2011). Four successive versions of GreenLab have been developed.

The first version of GreenLab, GL1, addressed deterministic crop plants and was initially applied to Maize (Guo et al. 2006). A second version, GL2, was developed to account for stochastic processes for growth, death, and branching patterns (Kang et al. 2008). It was initially developed for simple tree architecture, in which the time used for meristem extension is short and can be neglected compared to the time for biomass production and partitioning, and the processes can be decoupled. In the following versions, GL3 and GL4, corresponding respectively to GL1 and GL2, meristem activity was interactively linked to the supply/demand ratio, used to compute biomass thresholds (Mathieu et al. 2009) and extension probabilities. Although this led to a realistic pattern of tree growth, intra- and interannual shoot dynamics which include extension and rest periods were insufficiently accounted for.

\section{Mathematical model of meristem functioning}

The empirical and statistical distributions of phytomers inside a GU for various "seasonal" or "a-seasonal" trees show evidence of alternation between rest and extension periods of meristems in the shoot construction (de Reffye et al. 1991b; Jaeger and de Reffye 1992; Guedon et al. 2006; Galopin et al. 2010), which resemble "renewal processes". The renewal theory, related to time-Markov chains, enables to approximate the distribution of event arrivals in a given time period from the inter-arrival time distribution. 


\subsection{The renewal process}

According to Feller (1968), a renewal process is a stochastic model for events that occur randomly in time. The basic mathematical assumption is that the periods between successive arrivals, called renewal time (whose distribution is named the inter-arrival law), are independent and identically distributed. Given the mean $m$ and variance $v$ of renewal time, the number of events during time $T$ (whose distribution is named the counting law), is asymptotically normally distributed, and its mean $M$ and variance $V$ can be computed from renewal time as follows:

$\mathrm{M}=\frac{T}{m}, \quad \mathrm{~V}=\frac{T \cdot v}{m^{3}}$

Because of the convergence of the counting law towards a normal law, we can approximate this distribution by a binomial law $(N, b)$ with the above mean and variance. Accordingly, one gets:

$b=1-\frac{V}{M^{2}}, N=\frac{M}{b}, P(i)=C_{N}^{i} b^{i}(1-b)^{N-i}$

This binomial law can be considered as the result of a discrete Bernouilly process using step-by-step probability $b$. The counting law asymptotically depends only on the mean and variance of the inter-arrival law. The time $T$ can then be replaced by a virtual discrete time $N$. Moreover, assuming $V / M^{2}$ to be constant, the Bernouilly process does not depend on the scale chosen for the observation time $T$ : the scaling of the time axis does not affect the architecture or its variability, only the time taken by a plant to reach a specific architecture. An illustration of the above considerations is presented in Appendix 2, showing the distributions of events resulting from two different inter-arrival laws and the Monte Carlo simulation of a Bernouilly process.

\subsection{Modelling discrete development}

The time elapsed between the appearances of two successive phytomers can be regarded as renewal time. The counting variable, which is in this case the cumulated number of phytomers produced, theoretically converges to a normal distribution, which can be approximated by a binomial law for the discrete case. The continuous extension of a meristem during a period $T$ can be simulated by a Bernouilli process consisting of $N$ discrete steps of development. We shall name here "computing unit" (CU) the time between the appearances of two successive phytomers. At each CU, a phytomer appears with a probability $b$, so eventually the number of phytomers produced during $N$ CUs follows the binomial law $(N, b)$.
3.3 Modelling the development speed of an axis with continuous growth

In the case of continuous development, as observed on Eucalypt or Cypress (Barthélémy and Caraglio 2007), the speed of development can differ according to the physiological age of each axis. For instance, while the main stem can produce $x$ phytomers, higher-order axes may produce only $y<x$ phytomers in the same period. We call the ratio $w=y / x$ "rhythm ratio". Compared to probability $b$, the rhythm ratio modifies the average number of phytomers but has no effect on the variance.

\subsection{Modelling death of the meristem}

The meristem activity can stop at any moment according to a law of mortality. When mortality occurs at a given time, no phytomers are created and all the remaining CUs of an axis are filled with $0 \mathrm{~s}$. At each $\mathrm{CU}$, a fraction of the living population of meristems stops functioning. For continuous development, death of a meristem can take place at any CU. For rhythmic development, it is more realistic to consider death at the end of a growth unit. The proportion of surviving axes decreases according to axis age until their disappearance. In the case of sympodial growth, axes are considered as a succession of modules where death occurs at the end of every GU, and development of the axis continues with the subsequent GU.

\subsection{Modelling rhythmic development}

In the case of rhythmic development, two laws are associated respectively for the duration of meristem extension and duration of meristem rest between two successive GUs. The two periods alternate. The number of CUs between two successive GUs is the sum of the extension and rest periods, which can be regarded as renewal time. In regions with no marked seasons (a-seasonal), GUs can be produced at any time; for regions with marked seasons (seasonal), the renewal process of GU is rhythmic.

\subsection{Notion of axis of development}

The reference axis takes all the possible morphological differentiation stages of GUs according to their physiological age and is used in particular to simulate the different branching processes (de Reffye et al. 1991a; Barczi et al. 2008). In a similar way, the different aspects of meristem activity described above (3.2-3.5) can be represented by an "axis of development". It is made of a series of $1 \mathrm{~s}$ and $0 \mathrm{~s}$, which correspond to success and failure of phytomer production at each $\mathrm{CU}$. In the following section, examples of Monte Carlo simulations will be presented, representing the scalar product of series of either continuous or rhythmic development, and mortality process. 


\section{Detail of phytomer production within an axis}

\subsection{Shoots with continuous development}

$N$ is the number of CUs for axis development, $b$ is the probability for creating a phytomer at each CU. Let $c$ be the survival probability of a meristem at each CU. The whole population of axes at $\mathrm{CU} N$ can be separated into two populations: living axes (with probability $c^{N}$ ) and dead axes whose death can take place at any CU $i \quad(i<N)$ with probability $(1-c) c^{i}$. As a mixture of distributions, the probability of having $j$ phytomers during $N$ CUs is:

$$
\begin{aligned}
P(j)= & \sum_{i=0}^{N-1}(1-c) \cdot c^{i} \cdot C_{i}^{j} \cdot b^{j}(1-b)^{i-j} \\
& +c^{N} \cdot C_{N}^{j} \cdot b^{j}(1-b)^{N-j}
\end{aligned}
$$

The survival probability $c$ may vary, in which case at CU $i$, the probability of having a living axis is expressed as the product:

$f(i)=\prod_{j=1}^{i} c(j)$

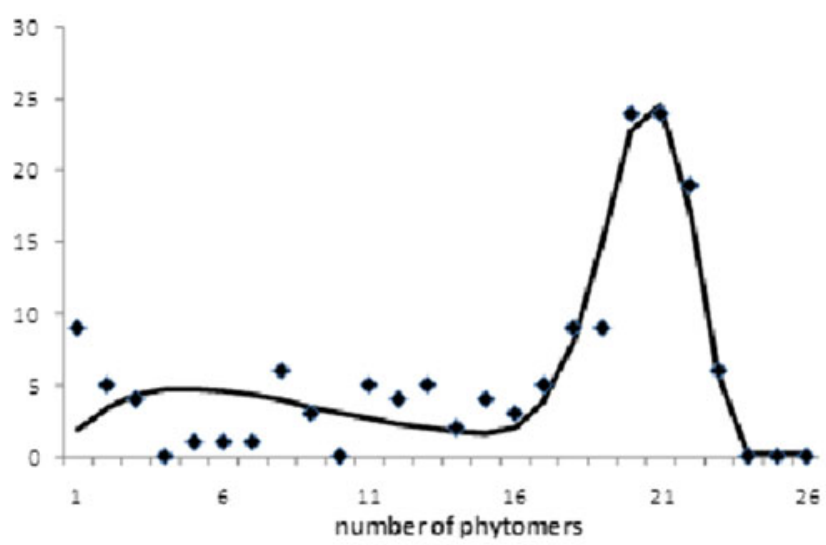

(a)

\subsection{Shoots with rhythmic development}

Let random variable $T_{f}$ be the functioning period in CUs, during which one can observe shoot extension, either by preformation or by neoformation. Let random variable $T_{r}$ be the rest period in CUs during which no visible event can be observed. As a first simplification for "a-seasonal" shoots, suppose the distributions of $T_{f}$ and $T_{r}$ are independent, with mean and variance being $m_{f}$, $v_{f}$, and $m_{r}, v_{r}$ respectively. As a result, the time between two successive GUs, $T_{g}$, is the sum of extension and rest periods, i.e., $T_{g}=T_{f}+T_{r}$, whose mean and variance are $m_{g}$ $=m_{f}+m_{r}, v_{g}=v_{f}+v_{r}$. According to the renewal law, the distribution of the number of GUs during a certain period follows a binomial law $\left(\mathrm{N}_{\mathrm{GU}}, \mathrm{b}_{\mathrm{GU}}\right)$.

\subsubsection{A-seasonal shoots}

In "a-seasonal" shoots, the axis of development alternates repetitively between extension and rest periods. For the extension period, we can fit a binomial law $\left(K_{f}\right.$, $b_{f}$ ) for its duration, whose mean and variance are $m_{f}=K_{f} \cdot b_{f}$

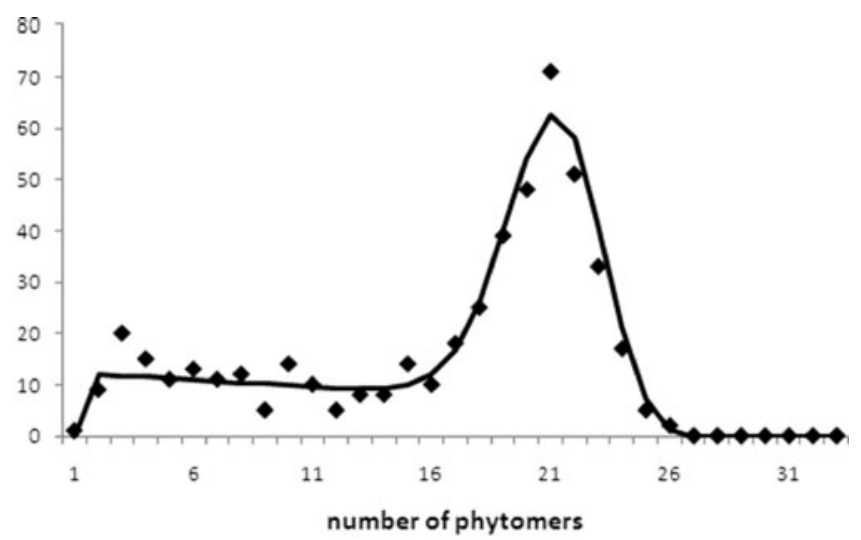

(b)

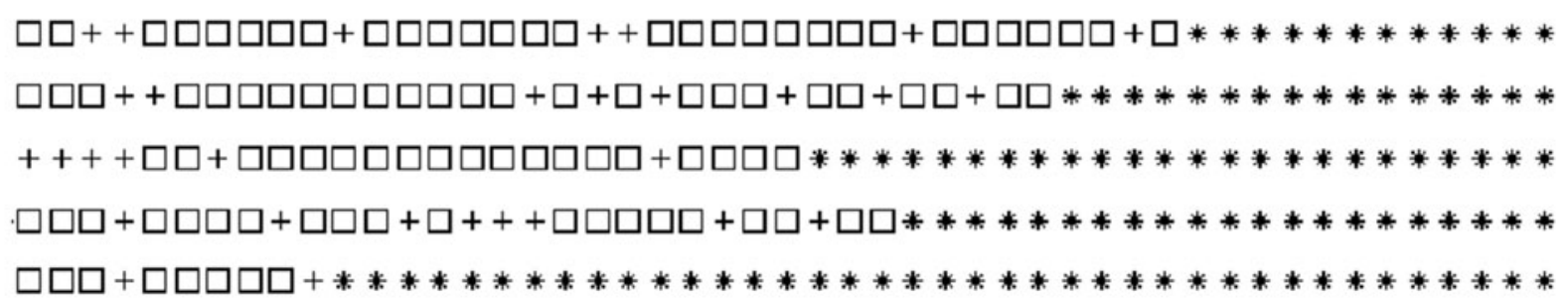

(c)

Fig. 1 Distribution of number of phytomers from a mixed development of Bernouilli process and death process: a Example of realization of this pattern in the internode distribution of bamboo (from Dabadie et al. 1991). b Monte Carlo simulation (dots) and theoretical computation (line), with $b=0.8$ and $c=0.97$. c Five simulated axes with continuous development, where each symbol represents a type of meristem activity at a given computing unit: idle (plus), creation of a phytomer (square), and dead meristem (asterisk) 
and $v_{f}=K_{f} \cdot b_{f}\left(1-b_{f}\right)$. If at each CU a phytomer is produced with probability $b$, then the cumulated number of phytomers in an annual axis will follow a compound distribution:

$P(j)=\sum_{i=0}^{N_{\mathrm{GU}}} P_{\mathrm{GU}}(i) \cdot C_{i \times K_{f}}^{j} b_{f}^{j}\left(1-b_{f}\right)^{i \times K_{f}-j}$

When the number of cycles takes the value $i$, there are $K_{f} \times i$ CUs available to produce the phytomers with probability $b_{f}$. For the distribution in CUs of the rest period, either a positive or negative binomial law is suitable depending on whether the variance of CUs is smaller or bigger than the mean.

\subsubsection{Seasonal shoots}

Here the development of axes is periodic, because in one year the number of CUs available for the develop- ment of the axes is fixed. Let $T_{T}$ be duration in CUs representing a year or the period between two favourable seasons. Development finishes when next $T_{g}$ is bigger than the remaining CUs in the year. Similar to the "aseasonal" case, the distribution of number of GUs in a year can be approximated by a binomial law according to the renewal theory, and the distribution of number of phytomers can be computed using a compound law.

\subsection{Shoots with pre- and neoformation}

The number of phytomers in a preformed GU follows a bell-shaped distribution and can be fitted by a positive binomial law. The number of phytomers in a neoformed GU can be fitted by a negative binomial law (de Reffye et al. 1991a). Preformation can be followed immediately by neoformation with a continuous extension until meristem breakdown. The number of phytomers in the annual shoot

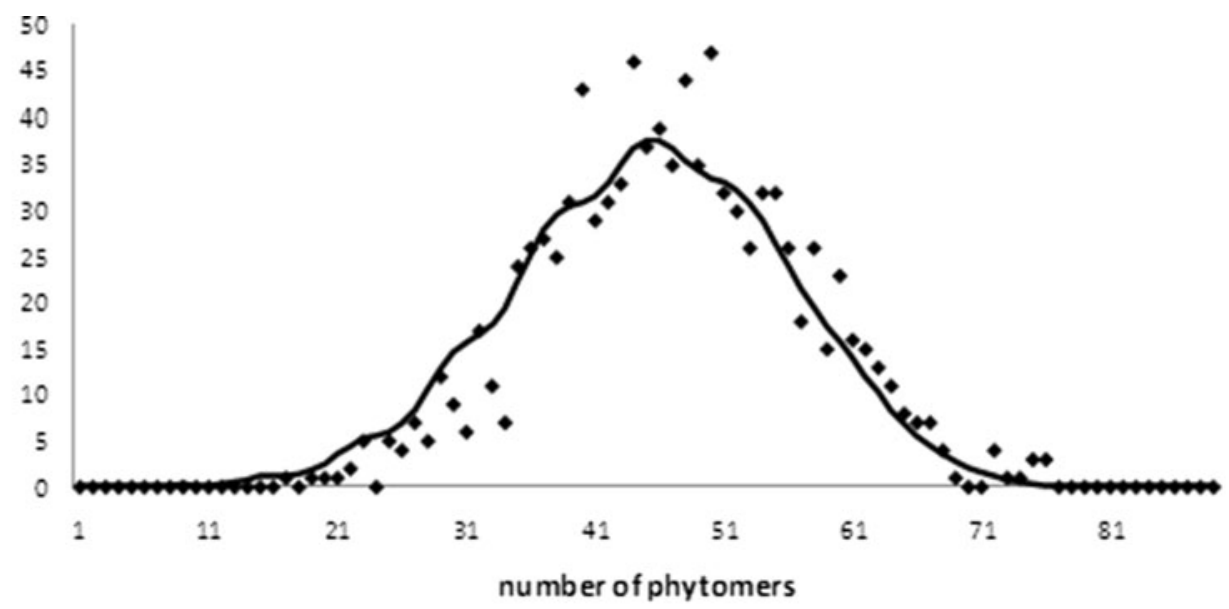

(a)

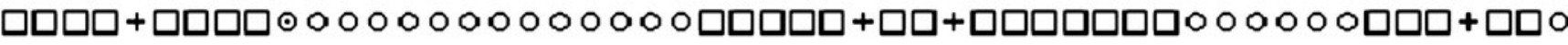

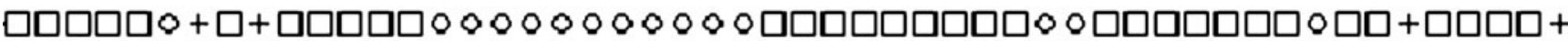

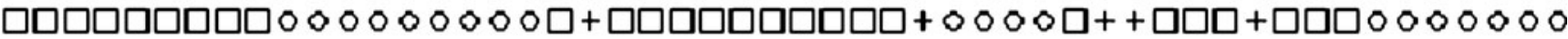

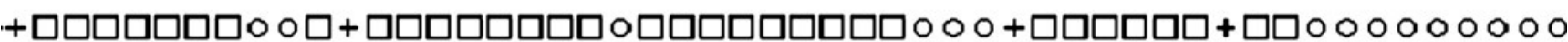

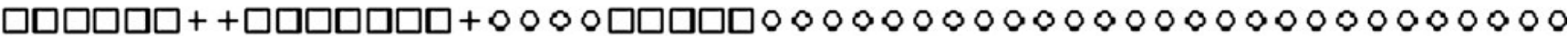

(b)

Fig. 2 Distribution of number of phytomers in "a-seasonal" annual shoots, as observed on Hevea (de Reffye et al. 1991b): a Monte Carlo simulation (dots) and theoretical computation (line). This distribution depends on the time for creating the phytomers of the growth unit (here a binomial law with $N=10$ and $b=0.8$ ), and the rest period between two growth units (which follows a negative binomial law with $K=1$ and $c=0.9)$. b Five simulated series of rhythmic shoot development, where each symbol represents a type of meristem activity at a given computing unit: idle (plus), creation of a phytomer (square) and rest period (circle). Parameters of the distributions are the same as above 
is then a convolution of the laws of pre- and neoformation (Guedon et al. 2006). This case is common in temperate trees, such as cherry, poplar, and elm (de Reffye et al. 1991a, b). The balance between the pre- and neoformation distributions may vary according to the plastic response of a tree to external factors and to the physiological age of the meristem: old meristems are less plastic and have no neoformation, while young meristems may undergo neoformation after having expressed their preformation (Barthélémy and Caraglio 2007).

(a)

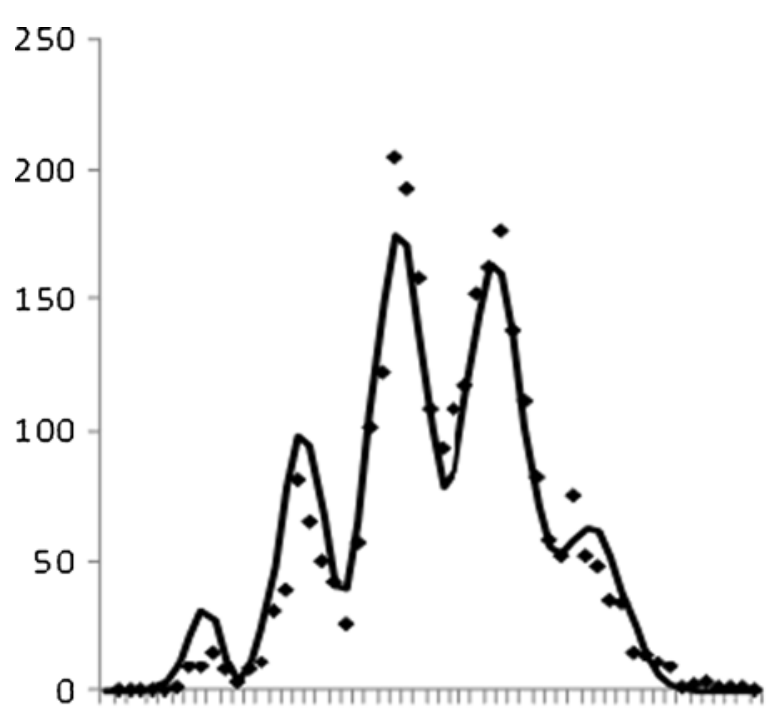

1591317212529333741454953

number of phytomers

\section{Simulation results}

To illustrate the theoretical considerations developed above, we have chosen examples of typical distributions representing the different types of shoot development.

\subsection{Continuous development}

Figure 1 shows (left, Figure 1a) an example of distribution of number of phytomers observed on a plant with continuous

(b)

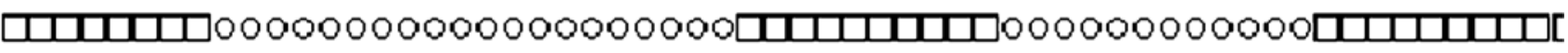

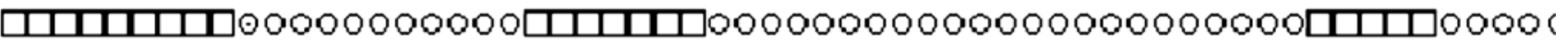

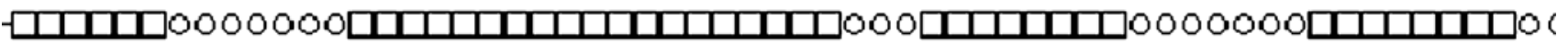

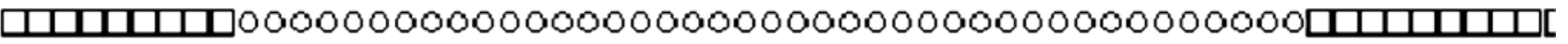

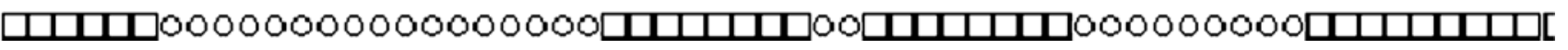

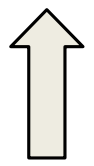

One Year
Fig. 3 Distribution of number of phytomers in a walnut annual shoot with polycyclism (based on Sabatier and Barthélémy 2001): a Monte Carlo simulation (dots) and theoretical computation (line). The extension period follows a binomial law $(10,0.8)$, and the rest period follows a negative binomial law $(1,0.9)$. Five growth units are visible. b Five simulated series of "seasonal" (temperate) shoot development with polycyclism, where each symbol represents a type of meristem activity at a given computing unit: creation of a phytomer (square) and rest period (circle).Parameters of the distributions are the same as above. The number of GUs is variable. The fourth line shows a single $\mathrm{GU}$ followed by a long period of rest 
development (bamboo, from Dabadie et al. 1991), and (right, Figure 1b) a distribution resulting from Monte Carlo simulation and theoretical computation from Eq. 1, with $b=0.8$ and $c=$ 0.95. Below (Figure 1c) are represented as an example of five simulated continuous development series of axes following this distribution. The distribution is a compound law between growth and death. The left tail of the distribution represents mainly dead axes, and the peak at the right are the surviving axes.

\subsection{Rhythmic development}

\subsubsection{A-seasonal shoots}

Based on observations on Hevea (de Reffye et al. 1991b), Figure 2a shows the distribution of number of phytomers

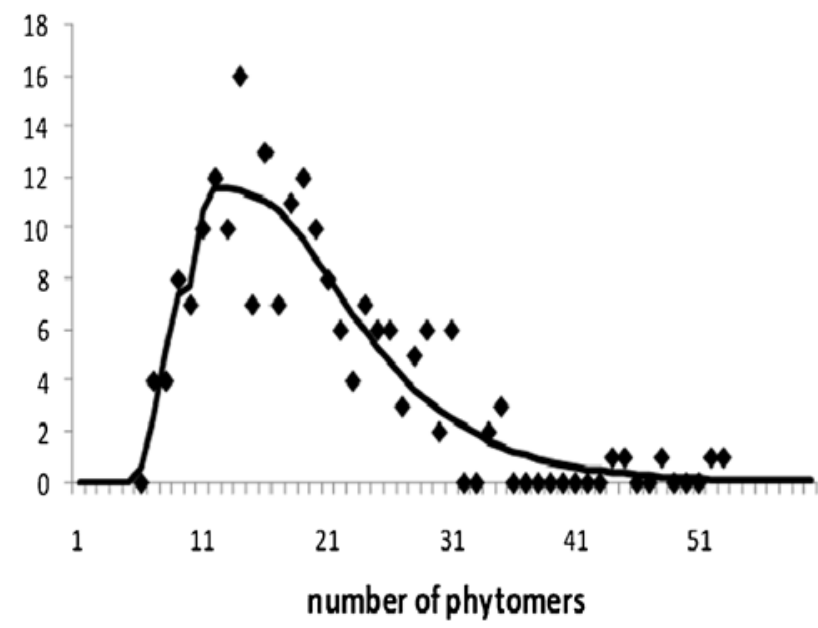

(a) (a) from an axis where $T_{f}$ follows a binomial distribution $(10,0.8)$ and $T_{r}$ follows a negative binomial distribution $(1,0.9)$. In the case of mortality, this distribution becomes a mixture of distributions as shown for continuous development. The distribution computed from the renewal theory (solid line) fits quite well the Monte Carlo simulation (dots). Figure $2 \mathrm{~b}$ shows five examples of simulated series of "a-seasonal" rhythmic shoot development, with alternation between extension (squares) and rest periods (circles).

\subsubsection{Seasonal shoots}

Figure $3 \mathrm{a}$ shows an example of distribution of number of GUs and phytomers for "seasonal" shoots with polyclism,

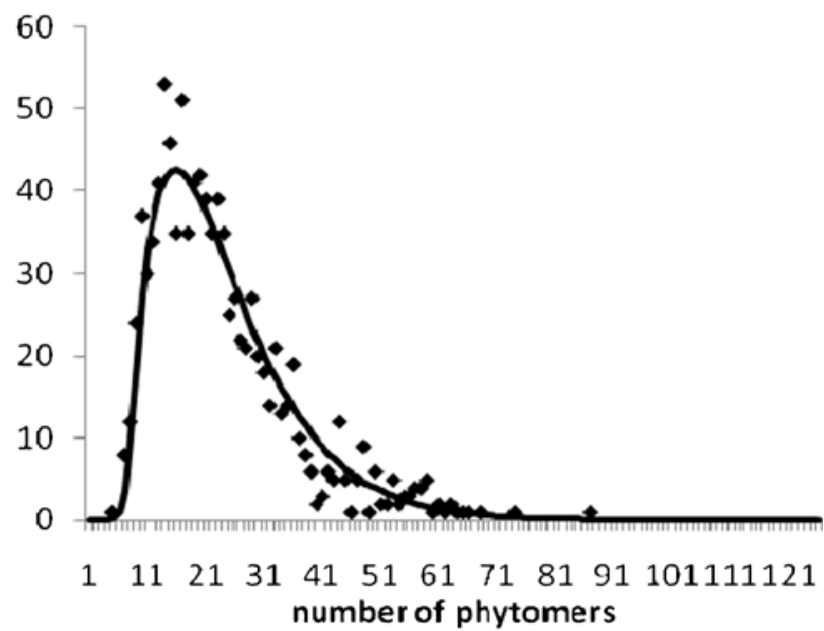

(b)
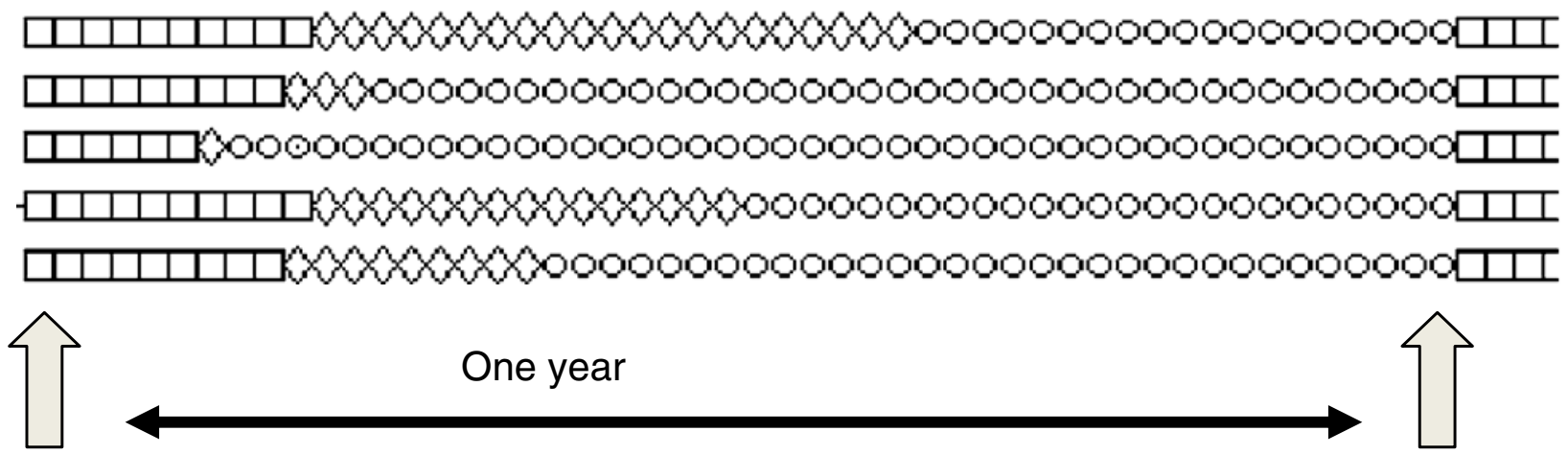

One year

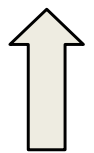

(c)

Fig. 4 Distribution of number of phytomers in an annual shoot exhibiting a mixture of preformation and neoformation. a Example of realization of this pattern in the annual shoot of Japanese elm (de Reffye et al. 1991a, b). b Monte Carlo simulation of 1,000 shoots, where the preformed part follows a binomial distribution $(N=15, b=$
$0.8)$ and the neoformed part follows a geometric distribution $(c=0.95)$. c Five simulated series of "seasonal" (temperate) shoot development with preformation and neoformation: extension of preformation part (square) is followed by neoformation (diamond) and ends with a rest period (circle). Parameters of the distributions are the same as above 
as can be observed on Walnut (Sabatier and Barthélémy 2001): the annual shoots are made of several GUs separated by rest periods. The renewal theory (solid line) fits the Monte Carlo simulation (dots). Figure $3 b$ shows five simulated series corresponding to shoots for "seasonal" trees with polycyclism. It can be seen that end of the year is a boundary, extension starts again in spring. In the monocyclic case, there is only one GU per year. The extension period $T_{f}$ used to expand the first GU is followed by a long rest period until the end of the year. The difference with Figure 2 is that the growth process is reset each year.

\subsection{Shoots with pre- and neoformation}

Figure 4 shows an example of preformation followed immediately by neoformation with a continuous extension until meristem breakdown, illustrated by Japanese Elm (a, de Reffye et al. 1991a) and Monte Carlo simulations (b and c). The preformed part (squares) is followed by the neoformed part (diamonds) generating a compound law for phytomer production. The breakdown of the meristem at the end of the extension period is followed by a rest period until the next year.

\subsection{Representation of whole tree architecture}

To visualize different processes of meristem functioning, two types of representation can be proposed for tree development: a chronological mode and a topological mode. In the chronological mode, a tree is a set of full and empty cells that represent the extension and rest periods of meristems: full cells represent the production of phytomers in the schedule of the tree development, while empty cells represent the rest period of the meristem. Figure 5a shows a simulated temperate tree structure following a Rauh model (according to Hallé et al. 1978) with rhythmic development and typical acrotony, as observed for most Pinus and Quercus species. Brachyblasts have a long rest period and short extension duration. The size of GUs depends on the stochastic laws of the meristem functioning. The time of appearance of organs and their duration are visible in the tree structure, which is linked to their role in the source and sink functioning. In the topological mode (Figure $5 b$ ), only the realized architecture is displayed and the time representation is included in the structure. This is the normal representation of the visible tree structure as seen in real trees.

Finally, a practical application of the simulations described above can be the realistic visualisation of trees for the needs of computer graphics. By adding to the above laws of meristem functioning, branching processes (from Kang et al. 2008), organ size, and geometrical rules (such as phyllotaxy, angles) measured on the plant, it is possible to simulate realistic stochastic tree architectures. Figure 6 is an example of two random simulations of a poplar tree, with annual rhythmic growth and meristems with pre- and neoformation, following the pattern of Figure 4.

\section{Discussion}

Architectural models, such as AMAPsim (Barczi et al. 2008), simulate correctly the dynamics of tree structure development, where the alternation of extension periods and rest periods of shoots, based on empirical observations, are reproduced in a satisfactory way. But they do not
Fig. 5 Simulation of a plant following the Rauh model. The Rauh model has a rhythmic growth. Growth units are preformed and show acrotony. The size of the GUs depends on physiological age. The physiological age of axillary branches increases along the growth unit from top to bottom. Each growth unit has its extension followed by a rest period until the end of the year. a The chronological mode shows the alternation of growth and rest periods of the meristems; $\mathbf{b}$ the topological mode shows the resulting stochastic topological structure

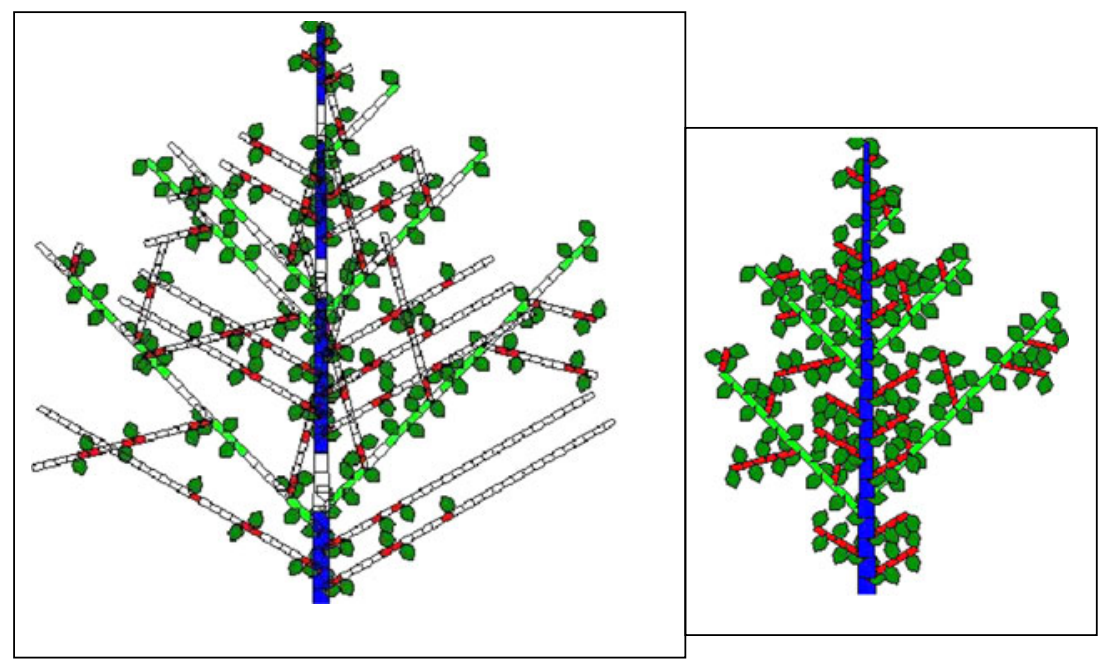

(a) (b) 


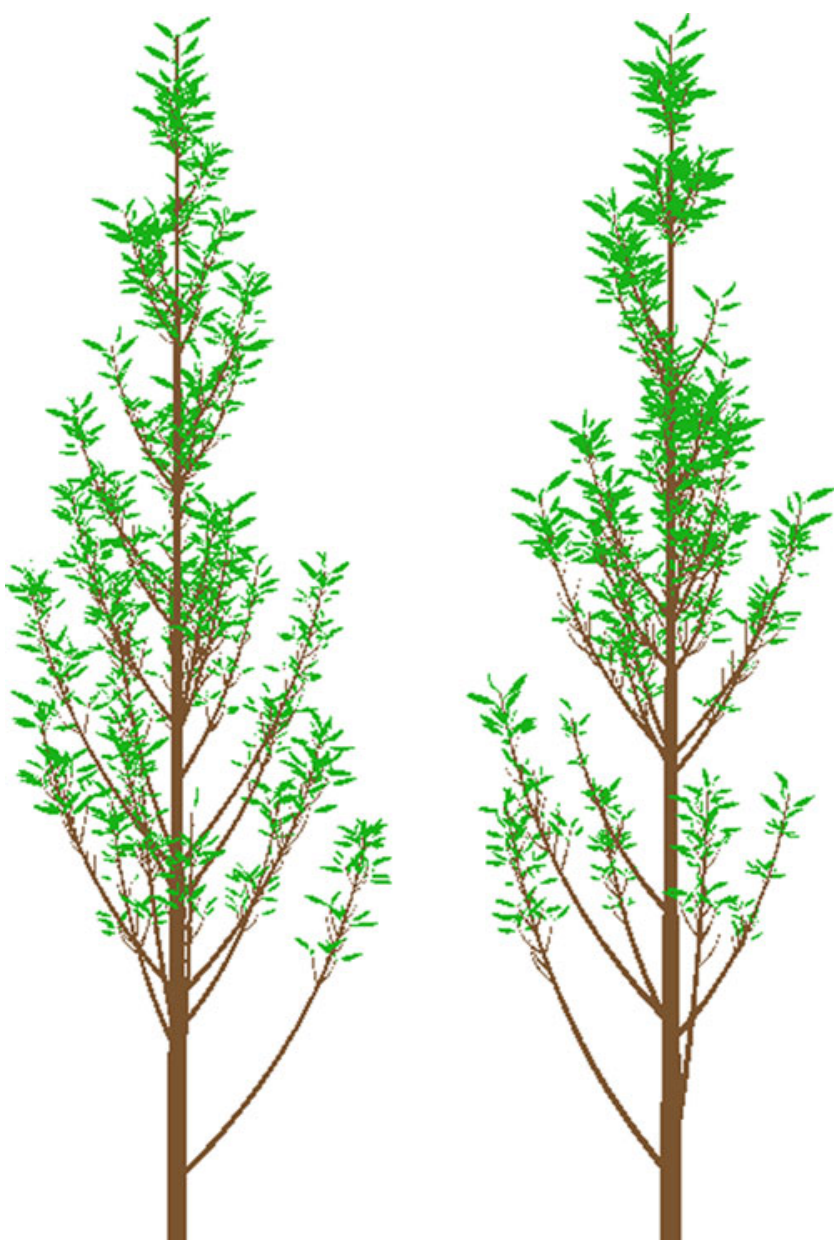

Fig. 6 Simulation of 9-year-old poplar tree structures, based on observations of Caraglio et al. (1990). Each year is divided into 60 CUs, and the trees are shown at CU 530. In total, there are three PAs. The GUs of PA 1 may contain two parts, a preformed part and a neoformed part. The extension period of each preformed part follows a binomial law $(15,1)$, while that of the neoformed part is $(20,0.8)$. At each $\mathrm{CU}$, the probability of producing a metamer is 0.8 . For axes of PA 2, which may also contain a preformed part and a neoformed part, the extension period of the preformed part follows a binomial law (10, $1)$, and the neoformed part $(10,0.8)$. At each $\mathrm{CU}$, the probability of producing a metamer is 0.8 . For PA 3, only preformed GUs is produced, whose extension period follows a binomial law $(5,1)$. At each $\mathrm{CU}$, the probability of producing a metamer is 0.6

consider ecophysiological functioning. To make further progress, with "functional-architectural plant models", it is necessary to link biomass production and partitioning with meristem functioning. For modelling trees with simple meristem functioning, where extension takes place in a short time before a long rest period, the architectural processes can be decoupled from the physiological processes. But for trees with more complex shoot development, photosynthesis carries on during the rest periods, woody rings develop, and all organs (leaves, internodes, fruits) continue their expansion. Therefore, tree development must be described numerically as an alternation of extension and rest periods, which was not the case until now. In FSPMs or FAPMs, which consider source and sink (or supply and demand) processes, this information is necessary to assess the plant demand.

The present work concerns only the development processes of single shoots, but it is destined to be applied to whole plant architecture, including branching. There are two ways of modelling plants in FSPMs or FAPMs, by simulation or by computation, which can lead to very different computation times for large plants, such as adult trees. Simulation of a plant follows the rules of development, which can be quite complex for some tree species, according to their "architectural model" (Hallé et al. 1978). The rules can be approached for example by growth grammars, such as those based on L-systems (Prusinkiewicz and Lindenmayer 1990), which simulate axis development. The entire structure is built and can be explored several times if biomass partitioning is required. Such simulation is proportional to the number of organs set in place by the meristems, and the time required for tree construction and/or for parameter estimation can increase dramatically with age.

The GreenLab model simulates tree architecture, which includes branching, using factorization of substructures and the notion of "axis of development". In the stochastic case, a collection of axes of development is constituted and chosen randomly to build substructures (Kang et al. 2003). The bigger the collection, the more the simulation can be approached by a pure Monte Carlo simulation, with a simulation time proportional to the collection size. Phytomer production in a tree structure can be obtained from simulation, but alternatively from recursive analytical equations. Kang et al. (2008) have proposed a mathematical formulation which applies a compound law of generating functions to compute recurrently the theoretical mean and variance of the number of organs in a plant structure. This analytical method provides fast and precise results, which facilitates model analysis as well as model calibration and validation with real plants. The present work is an additional contribution to such mathematical formalism, which decreases computation time and allows using inverse methods such as nonlinear least squares to assess the source and sink parameters.

\section{Conclusion}

We propose here a theoretical improvement of former versions of the FAPM GreenLab that prepares a further, more generic implementation for tree growth. Based upon the renewal theory, theoretical distributions of number of organs in an annual shoot have been obtained. Further development of this work is necessary to obtain the 
Fig. 7 Illustration of the renewal theory. Two different inter-arrival laws, with the same mean and variance, exhibit similar patterns: 1 binomial law $(N=9, b=0.66), 2$ shifted geometric law $(d=5$, $c=0.5)$. They can be approximated with a binomial law whose parameters are deduced from the above parameters $(N=18, b=0.94)$
Counting Laws simulated compared to Binomial law

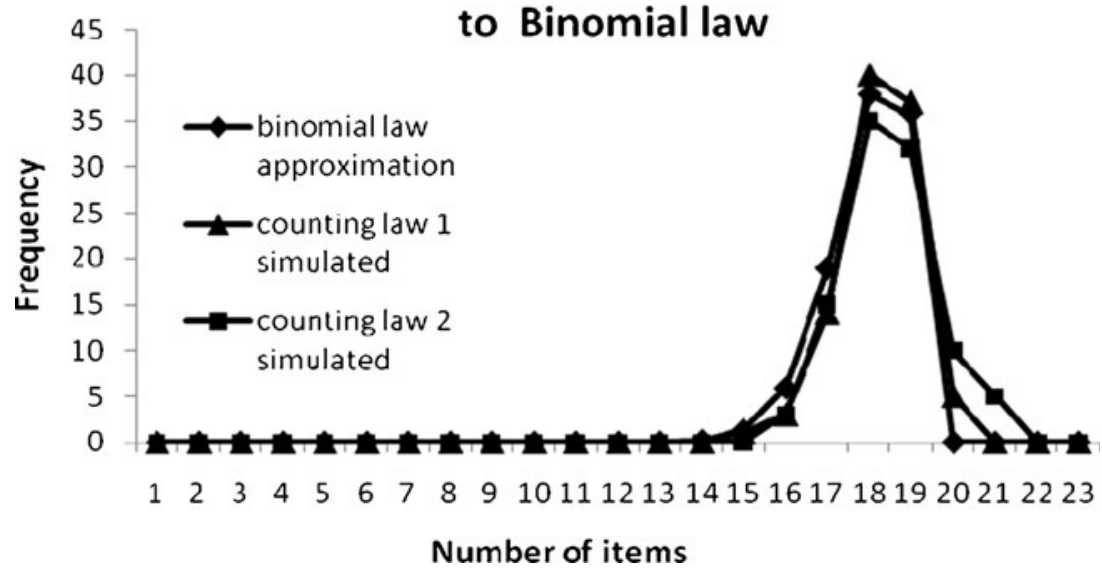

analytical mean and variance of organ production at plant level as was done previously for a simpler stochastic case (Kang et al. 2008). In addition, the current work does not consider the feedback between development and growth as was described by Mathieu et al. (2008): the schedule of development remains forced even if it can follow step-bystep a change of meristem activity along time. Further work will be required to address the question of the feedback of biomass production on meristem functioning.

Acknowledgments This work was undertaken as a collaborative work within the LIAMA (the Sino-French laboratory for computer science, automation and applied mathematics) with funding from the Natural Science Foundation of China (grant numbers 31170670), Zhejiang Tengtou Landscape Co. LTD., CIRAD, and INRA.

\section{Appendix 1: the botanical bases (after Barthélémy and Caraglio 2007)}

Meristem functioning of the annual shoot

\section{Continuous vs. rhythmic development}

Apical meristems contribute to axis development by adding new phytomers step by step. The functioning can be continuous or rhythmic. In the continuous case, phytomers are added one by one without a significant rest period. The cumulated number of metamers in an axis is generally proportional to the daily sum of temperatures ("thermal time"). Many plants have a development following this pattern (tomato, maize, cotton, coffee). In the rhythmic case, the meristem alternates between extension periods and rest periods; as a result, an annual shoot is made of GUs that are sets of phytomers built during the same extension period. The GU can be issued from preformation or neoformation (see Sec. 4.2). During the rest period of the meristem, generally a bud is built containing embryos of future phytomers.
Monocyclic vs. polycyclic case

Rhythmic development can be monocyclic or polycyclic. In the monocyclic case, only one GU is produced each year. The extension of GUs (often from preformation) usually ends in spring, and the rest period will complete the year. In the polycyclic case, the annual shoot can be made of several GUs.

Seasonal vs. a-seasonal development

There can be two different modes in rhythmic development. In regions with a marked seasonal difference (temperate regions or regions with marked dry and/or rainy seasons), the development of the axes is periodic: each year, the meristem extension ends by a rest period and then starts again (in spring). The development of all axes is synchronized. Inside a year, the development can be monocyclic or polycyclic, depending on the climate and mainly temperature: Pinus sylvestris is monocyclic in Finland, whereas it is polycyclic in Southern France. So there are rhythms at two different time scales: inside a year or between years. In regions with no marked seasonal difference (tropical or equatorial, named here $a$ seasonal), meristem functioning can last all year round with or without a rest period, so there is no boundary of yearly development. Polycyclism can be indefinite and flushes can be asynchronous according to physiological age of branches.

\section{Preformation vs neoformation}

Preformation is common in the case of rhythmic development when a bud is formed during a rest period, as observed in beech or poplar. The flush, or the simultaneous extension of all organs in a bud, generates a GU named preformed part, which can be followed by several months before the next flush. On the other hand, continuous functioning of meristems gives birth to a neoformed part. For some particular tree architectures, such 
as elm or poplar, it can take place immediately after the extension of the preformed part.

\section{Plant architecture}

The analysis of tree architecture according to botanical considerations was initiated by Hallé et al. (1978). The entire plant architecture is under control of the meristem functioning as introduced above. The branching pattern can be monopodial or sympodial. Axes with the same development history can adopt different kinds of morphology and behaviour, being plagiotropic or orthotropic. The combination of different botanical traits leads to the notions of architectural model and reiteration. Barthélémy et al. (1989) refined the notion of architectural model into that of "architectural unit" that describes the type of axis for each branching order, which is the support of tree organization. The level of differentiation of the terminal meristem that gives birth to different types of axes is named "physiological age". Physiological age accounts for the main gradients of axis organization in the tree architecture. These changes in meristem functioning can be represented by the "reference axis", a single theoretical graduated axis, which successively takes all the possible morphological differentiation stages of GUs according to their physiological age (de Reffye et al. 1991a, 1995; Barczi et al. 2008).

\section{Appendix 2: illustration of the renewal theory}

As an illustration of the use of the renewal theory applied to meristem functioning, Figure 7 shows the distributions resulting from two different inter-arrival laws, a binomial and a shifted geometric law, with the same mean and variance: $m=6, v=2$.

Law 1 is a binomial law with $N=9$ and $b=0.67$;

This gives: $m_{1}=9 \times 0.67 \approx 6 ; v_{1}=9 \times 0.67 \times(1-0.67) \approx 2$

Law 2 is a shifted geometric law with $d=5$ and $c=0.5$;

This gives: $m_{2}=5+0.5 /(1-0.5)=6 ; v_{2}=0.5 /(1-0.5)^{2}=2$

From the renewal theory, we can consider that the normal law at the time $T$ with mean $M=\frac{T}{m}$, and variance $V=\frac{T . v}{m^{3}}$ will give a good approximation of the counting law. Moreover, it can be replaced by a binomial law $(N, b)$ whose parameters are deduced from Eq. 2. This gives $b=1-$ $v / m^{2}=1-2 / 6^{2}=0.94$

If we run both renewal processes using Monte Carlo simulations for a period $T=100$, the corresponding Binomial law is $N=100 /(6 \times 0.94) \approx 18, b=0.94$

We can observe that the results from the simulation and those computed from the counting laws fit very closely. These results show that the prediction of the renewal theory are very satisfactory and the choice of the Bernouilly process to compute the counting law as a binomial law appears as a relevant simplification.

\section{References}

Barczi JF, Rey H, Caraglio Y, de Reffye P, Barthélémy D, Dong Q, Fourcaud T (2008) AMAP-Sim: a structural whole-plant simulator based on botanical knowledge and designed to host external functional models. Ann Bot 101:1125-1138

Barthélémy D, Caraglio Y (2007) Plant architecture: a dynamic, multilevel and comprehensive approach to plant form, structure and ontogeny. Ann Bot 99:375-407

Barthélémy D, Edelin C, Hallé F (1989) Architectural concepts for tropical trees. In: Holm-Nielsen LB, Balslev H (eds) Tropical forests: botanical dynamics, speciation and diversity. Academic, London, pp 89-100

Biliouris D, Van der Zande D, Verstraeten WW, Muys B, Coppin P (2009) Assessing the impact of canopy structure simplification in common multilayer models on irradiance absorption estimates of measured and virtually created Fagus silvatica (L.) stands. Remote Sens 1:1009-1027

Bosanac B, Zanchi P (2002) Onyx tree conifer user's manual, version 5.1. Onyx Computing Inc, Cambridge

Caraglio Y, Elguero E, Mialet I, Rey H (1990) Le Peuplier: modélisation et simulation de son architecture. CIRAD/IDF report, Montpellier, FR

Dabadie P, de Reffye P, Dinouard P (1991) Modelling bamboo growth and architecture: Phyllostachys viridi-glaucescens A \& C Rivière. J Am Bamboo Soc 8:65-79

De Jong T, Da Silva D (2010) FSPM2010: proceedings of the 6th international workshop on functional-structural plant models. Univ California, Davis

de Reffye P, Dinouard P, Barthélémy D (1991a) Modélisation et simulation de l'architecture de l'Orme du Japon Zelkova serrata (Thunb) Makino (Ulmaceae): la notion d'axe de référence. Naturalia Monspeliensia Sp Issue:251-266

de Reffye P, Elguero E, Costes E (1991b) Growth units construction in trees: a stochastic approach. Acta Biotheor 39:325-342

de Reffye P, Houllier F, Blaise F, Barthélémy D, Dauzat J, Auclair D (1995) A model simulating above- and below-ground tree architecture with agroforestry applications. Agrofor Syst 30:175-197

de Reffye P, Heuvelink E, Barthélémy D, Cournède PH (2008) Plant growth models. In: Jorgensen SE, Fath BD (eds) Encyclopedia of ecology, vol 4. Elsevier, Amsterdam, pp 2824-2838

Deussen O, Lintermann B (2005) Digital design of nature: computer generated plants and organics. Springer, Berlin

Feller W (1968) An introduction to probability theory and its applications, vol. 1, 3rd edn. Wiley, New York

Feng L, de Reffye P, Dreyfus P, Auclair D (2012) Connecting an architectural plant model to a forest stand dynamics modelapplication to Austrian black pine stand visualization. Ann For Sci. doi:10.1007/s13595-011-0144-5

Galopin G, Mauget JC, Morel P (2010) Morphogenetic analysis of the phenotypic variability of the architectural unit of Hydrangea macrophylla. Ann For Sci 67. doi:10.1051/forest/2009115

Godin C (2000) Representing and encoding plant architecture: a review. Ann For Sci 57:413-438

Griffon S, Nespoulous A, Cheylan JP, Marty P, Auclair D (2011) Virtual reality for cultural landscape visualization. Virtual Reality. doi:0.1007/s10055-010-0160-z

Guedon Y, Puntieri J, Sabatier S, Barthélémy D (2006) Relative extents of preformation and neoformation in tree shoots: analysis by a deconvolution method. Ann Bot 98:835-844 
Guo Y, Ma Y, Zhan Z, Li B, Dingkuhn M, Luquet D, de Reffye P (2006) Parameter optimization and field validation of the functional-structural model GREENLAB for maize. Ann Bot 97:217-230

Hallé F, Oldeman RAA, Tomlinson PB (1978) Tropical trees and forests. Springer, Berlin

Jaeger M, de Reffye P (1992) Basic concepts of computer simulation of plant growth. J Biosci 17:275-291

Kang MZ, de Reffye P, Barczi JF, Hu BG, Houllier F (2003) Stochastic $3 \mathrm{D}$ tree simulation using substructure instancing. In: $\mathrm{Hu}$ BG, Jaeger M (eds) Plant growth modelling and applications. Tsinghua University Press, Beijing, pp 154-168

Kang MZ, Cournède PH, de Reffye P, Auclair D, Hu BG (2008) Analytical study of a stochastic plant growth model: application to the GreenLab model. Math Comput Simul 78:5775

Kimmins JP, Blanco JA, Seely B, Welham C, Scoullar K (2008) Complexity in modelling forest ecosystems: how much is enough? For Ecol Manag 256:1646-1658

Kohyama T, Ojima DS, Pitelka LF (2005) Forest ecosystems and environments: scaling up from shoot module to watershed. Springer, Berlin

Kurth W (1994) Morphological models of plant growth: possibilities and ecological relevance. Ecol Model 75-76:299-308

Le Roux X, Lacointe A, Escobar-Guttierez A, Le Dizes S (2001) Carbon-based models of individual tree growth: a critical appraisal. Ann For Sci 58:469-506

Mäkelä A, Landsberg J, Ek AR et al (2000) Process-based models for forest ecosystem management: current state of the art and challenges for practical implementation. Tree Physiol 20:289-298

Mathieu A, Cournède PH, Barthélémy D, de Reffye P (2008) Rhythms and alternating patterns in plants as emergent properties of a model of interaction between development and functioning. Ann Bot 101:1233-1242
Mathieu A, Cournède PH, Letort V, Barthélémy D, de Reffye P (2009) A dynamic model of plant growth with interactions between development and functional mechanisms to study plant structural plasticity related to trophic competition. Ann Bot 103:1173-1186

Melson SL, Harmon ME, Fried JS, Domingo JB (2011) Estimates of live-tree carbon stores in the Pacific Northwest are sensitive to model selection. Carbon Balance Manag 6:2

Parsons RA, Mell WE, McCauley P (2011) Linking 3D spatial models of fuels and fire: effects of spatial heterogeneity on fire behavior. Ecol Model 222:679-691

Perttunen J, Sievänen R, Nikinmaa E, Salminen H, Saarenmaa H, Väkevä J (1996) LIGNUM: a tree model based on simple structural units. Ann Bot 77:87-98

Pretzsch H (2009) Forest dynamics, growth and yield. Springer, Berlin

Prusinkiewicz P, Lindenmayer A (1990) The algorithmic beauty of plants. Springer, New York

Sabatier S, Barthélémy D (2001) Annual shoot morphology and architecture in Persian Walnut, Juglans regia L. (Juglandaceae). Acta Hortic 544:255-264

Vos J, Marcelis LFM, Evers JB (2007) Functional-structural plant modelling in crop production-adding a dimension. In: Vos $\mathrm{J}$, Marcelis LFM, de Visser PHB, Struik PC, Evers JB (eds) Functional-structural plant modelling in crop production. Springer, Amsterdam, pp 1-12

Vos J, Evers JB, Buck-Sorlin GH, Andrieu B, Chelle M, de Visser PHB (2010) Functional-structural plant modelling: a new versatile tool in crop science. J Exp Bot 61:2101-2115

Wang F, Kang MZ, Lu Q, Letort V, Han H, Guo Y, de Reffye P, Li B (2011) A stochastic model of tree architecture and biomass partitioning: application to Mongolian Scots pines. Ann Bot 107:781-792

Yan HP, Kang MZ, de Reffye P, Dingkuhn M (2004) A dynamic, architectural plant model simulating resource-dependent growth. Ann Bot 93:591-602 